\title{
The workload of a psychiatric registrar in a mental handicap hospital-implications for training and community care
}

\author{
Glyn Jones, Registrar; and Mike KerR, Lecturer, Department of Psychological \\ Medicine, Ely Hospital, Cardiff CF5 5XE
}

The widespread move to community care for people with a learning disability has caused a change in roles for both the psychiatrist and the general practitioner. The general practitioner is now widely recognised as the primary medical carer for people with a learning disability (Howells, 1991), with the psychiatrist providing specialist support.

Although research has shown deficiencies in the care of people with a learning disability in the community (Howells, 1986), we do not as yet know what specialised skills, if any, the GP will need in his new role, though it is certain that in future primary care will need more specific organisation to provide care for the learning disabled (Cumella et al, 1992).

Current psychiatric training in learning disability often requires the trainee to provide "primary care" to hospital wards - that is providing basic medical care on a day to day basis. The relevance of this to the trainee's future as a potential consultant psychiatrist is unknown. This study was designed to help answer the following questions: does the GP need specialised skills to care for people with a learning disability? And does providing "primary care" to in-patients in a mental handicap hospital provide relevant training for the psychiatric trainee?

\section{The study}

As part of a six month assignment in learning disabilities the psychiatric trainee covers the health needs of several wards in a mental handicap hospital. This is one aspect of his training which also includes out-patient and neuro-psychiatric experience.

A prospective analysis of all contacts (either in person or telephone) was undertaken in the months of April and June 1992 to three of the wards. The wards were representative of the trainee's workload and also felt to be typical of those residents currently being resettled into the community. Two separate months were chosen to allow for seasonal differences.

Every doctor contact with resident or staff was recorded on a data collection form. Data were collected on urgency of call, time taken, diagnosis, further investigations, treatment and need of specialist referral. All out of hour calls were similarly recorded.

\section{Findings}

The wards

The three wards consisted of 60 residents. Of these, $41(68 \%)$ were male and $19(32 \%)$ were female. The severity of learning disability by ICD-9 was severe $37(62 \%)$, moderate $16(27 \%)$, and mild $7(11 \%)$. The age range was male $21-88$ (mean 60 ) and female 25-87 (mean 55).

\section{Contacts}

A total of 103 contacts were made in the two months averaging 1.7 per day. However the contacts were with only $33(55 \%)$ of the residents. Further analysis revealed that $28(27 \%)$ of these contacts were to just four residents, three of whom were seen for psychiatric/behavioural problems. Only five $(5 \%)$ visits were out of hours.

\section{Reason for call}

The reason for each contact - by diagnostic category - is shown in Fig. 1. The range of morbidity is compared to that typically seen by a GP (Morrell, 1991). The major difference in distribution is the increase in contacts for psychiatric/behavioural reasons.

\section{Action taken}

In nine $(9 \%)$ cases advice alone was sufficient. A physical examination was performed in $54(52 \%)$ of contacts. Medication was prescribed in $28(27 \%)$ of cases. Further investigations were performed in $21(20 \%)$. These included anticonvulsant levels 4 , other bloods 11 , mid stream urine 3 , stool culture, and chest $X$-ray in 2 . Seven residents $(11 \%)$ were referred for a second opinion, two orthopaedic, four psychiatric (in these further advice was sought from the consultant or senior registrar) and one dental. 


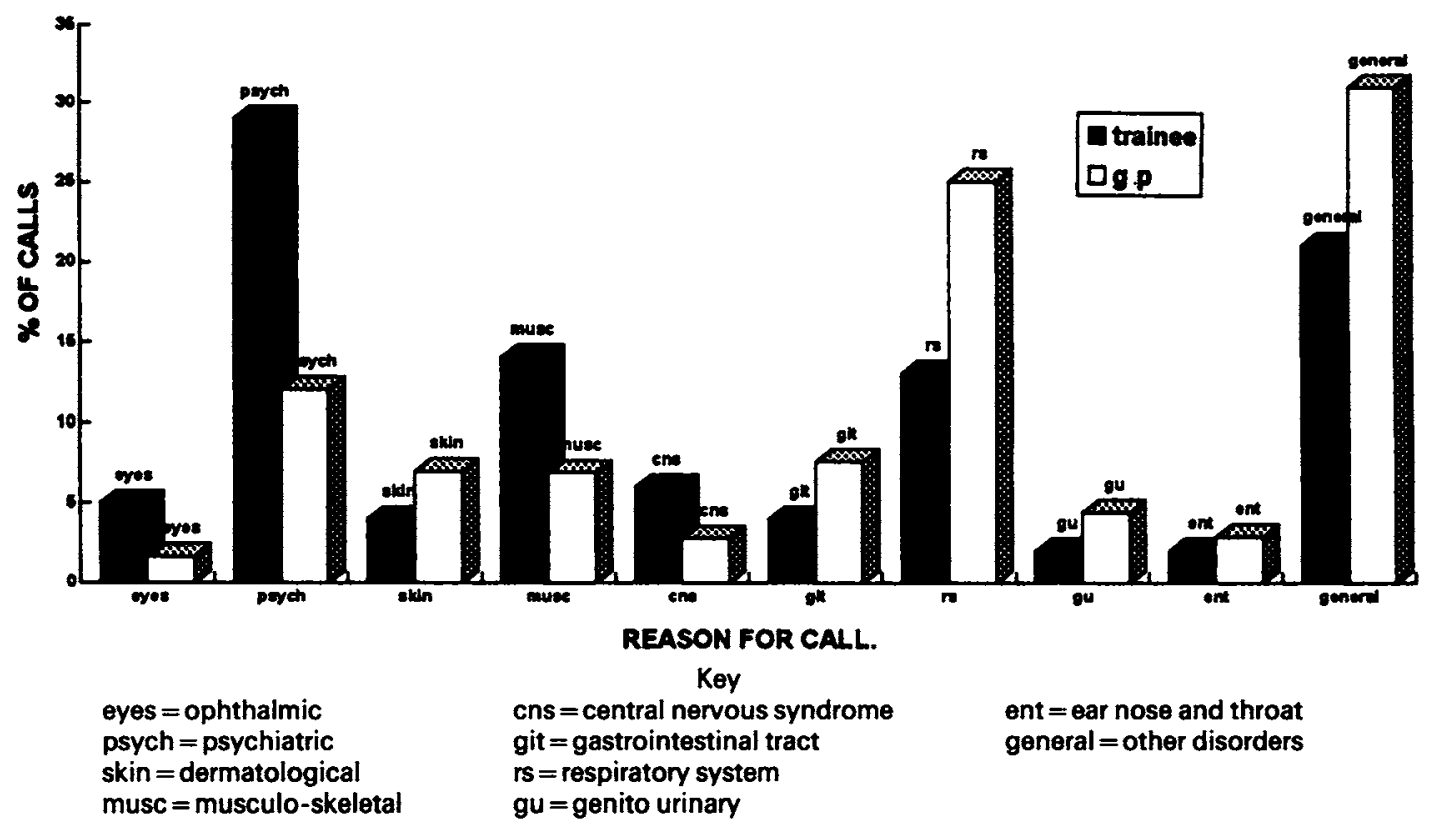

FIG. 1. A comparison of morbidity seen by psychiatric registrar with typical general practice morbidity.

\section{Time taken}

The total time taken was up to one minute in $11(11 \%)$ cases, one to five minutes in $42(41 \%)$ cases, five to 15 minutes in $31(30 \%)$ cases and 15 minutes to one hour in $19(18 \%)$ cases. It is important to note that 15 of the 19 contacts which took 15 minutes to one hour were for psychiatric/behavioural problems.

\section{Comment}

A criticism of this study is how relevant is it to compare current in-patient practices with future community care? We do not, of course, know the answer to this; however, the trends seen in hospital are possible indicators of future community morbidity. The data, although limited, do have implications for community care and psychiatric training.

The data complement the opinion of Howells (1986) that the medical needs of adults with a learning disability do not place a greater burden on the GP than other members of the public. The call-out rate per individual was low and of such an urgency that in most cases the individual could have attended surgery. We do not know how the "call-out" rate in hospital reflects that in the community with its differing profile of staff support affecting tolerance of health and behavioural problems. However, in this study a very few individuals accounted for a large amount of the trainee's workload. These residents had severe chronic psychiatric/behavioural problems needing a complex multidisciplinary approach. The management of such individuals by the GP alone would not be possible. Therefore services must exist in the community to deal with these few complex individuals. Any "special skills" needed by the GP are likely to be in the field of behavioural management and working with the multidisciplinary team. How well does providing this "primary medical care" train the psychiatrist? It would certainly seem that the trainee's workload involves both management of specialised psychiatric/behavioural problems as well as providing "primary care" experience. This would seem to confirm that the existing training is relevant to the psychiatrist's needs - but its relevance to the psychiatrist's future needs must be kept under review.

\section{Acknowledgement}

We are grateful for the advice given in the writing of this paper by Professor W. I. Fraser.

\section{References}

Cumella, S., Corbett, J. A., Clarke, D. \& Smtri, B. (1992) Primary health care for people with a learning disability. Mental Handicap, 20, 123-125.

HoweLLs, G. (1986) Are the needs of mentally handicaped adults being met? Journal of The Royal College of General Practitioners, 36, 449-453.

(1991) Mental handicap-care in the community. Journal of The Royal College of General Practitioners, 1 , 2-4.

MORRELl, D. (1991) The Art of General Practice. Oxford Medical Publications. p. 75. 